\title{
INFLUENCIA DE LA LLUVIA ANTECEDENTE Y LA CONDUCTIVIDAD HIDRÁULICA EN LA OCURRENCIA DE DESLIZAMIENTOS DETONADOS POR LLUVIAS UTILIZANDO EL MODELO SHIA_LANDSLIDE
}

\author{
- Edier Vicente AristizÁbal Giraldo ${ }^{1}$ \\ JAIME IGNACIO VÉLEZ UPEGUI ${ }^{2}$ \\ HERnán EduARdo Martínez CARVAJAL ${ }^{1,2}$
}

\section{RESUMEN}

Las pérdidas económicas y humanas por movimientos en masa ocurren cada año en todo el mundo; sin embargo el impacto varía considerablemente debido a las condiciones geológicas locales y vulnerabilidad socioeconómica. Aunque la ocurrencia de movimientos en masa ha golpeado Los Andes colombianos, pocos estudios que consideren la lluvia como factor detonante se han realizado. SHIA_Landslide (Simulación Hidrológica Abierta para deslizamientos detonados por lluvia) es un modelo conceptual y de base física para analizar los procesos de deslizamientos superficiales mediante la incorporación de un modelo hidrológico de tanques y distribuido que incluye el almacenamiento de agua en el suelo, acoplado con un análisis clásico de estabilidad de talud infinito en condiciones saturadas. En este trabajo se evalúa la influencia de la lluvia antecedente y conductividad hidráulica de los deslizamientos detonados por lluvias utilizando el modelo SHIA_Landslide. Los resultados obtenidos en este estudio son similares a los obtenidos por diferentes autores en la literatura. Los suelos con altos valores de conductividad solo necesitan lluvias cortas e intensas para fallar, y las condiciones de lluvia antecedentes no juegan un papel importante para este tipo de suelos.

PALABRAS CLAVE: Modelos físicos; deslizamientos detonados por lluvias; SHIA_Landslide.

\section{INFLUENCES OF ANTECEDENT RAINFALL AND HYDRAULIC CONDUCTIVITY ON LANDSLIDES TRIGGERED BY RAINFALL OCCURRENCE USING THE MODEL SHIA_LANDSLIDE}

\section{ABSTRACT}

Human and economic losses generated by landslides occur every year in all countries; however, the impact of landslides varies considerably according to local geological conditions and socio-economic vulnerability. Although

1 PhD, Departamento de Geociencias y Medioambiente, Universidad Nacional de Colombia, Medellín, Colombia

2 PhD, Departamento de Ingeniería Civil y Ambiental, Facultad de Tecnología, Universidad de Brasilia, Brasilia, Brasil

Autor de correspondencia: Aristizábal, E. (Edier): Carrera 80 No 65-223. Teléfono: 42551 97. Correo electrónico: evaristizabalg@unal.edu.co
Historia del artículo:

Artículo recibido: 13-IV-2016 / Aprobado: 16-XI-2016

Disponible online: 30 de febrero de 2017

Discusión abierta hasta abril de 2018 
the occurrence of landslides has impacted the Colombian Andes for a long time, few studies considering rainfall as a triggering factor have been carried out in our country. SHIA_Landslide (Open and Distributed Hydrological Simulation \& Landslides) is a conceptual and physically based model to analyze shallow landslide processes by incorporating a comprehensive distributed hydrological tank model that includes water storage in the soil coupled with a classical analysis of infinite-slope stability under saturated conditions. This paper evaluates the influence of antecedent rainfall and hydraulic conductivity on landslides triggered by rainfall using the SHIA_Landslide model. The results obtained in this study are similar to those presented by different authors in the literature. Soils of highconductivity values only need short and intense rainstorms for failure, and the antecedent rainfall conditions donot play an important role for these kinds of soils.

KEYWORDS: Physical models; Landslide triggered by rainfall; SHIA_Landslide.

\section{INFLUÊNCIAS DA CHUVA ANTECEDENTE E A CONDUTIVIDADE HIDRÁULICA NA OCORRÊNCIA DE DESLIZAMENTOS TETONADOS PELAS CHUVAS UTILIZANDO O MODELO SHIA_LANDSLIDE}

\section{RESUMO}

As perdas humanas e econômicas geradas por deslizamentos de terra ocorrem todos os anos em todos os países; no entanto, o impacto de deslizamentos de terra varia consideravelmente devido ás condições geológicas locais e a vulnerabilidade sócio-econômica. Embora a ocorrência de deslizamentos de terra têm impactado os Andes colombianos, poucos estudos que considerem a chuva como fator detonante tem- se realizado. SHIA_Landslide (Simulação Hidrológica aberta para deslizamentos detonados pela chuva) é um modelo conceitual e de base física para analisar os processos de deslizamentos superficiais pela incorporação de um modelo hidrológico de tanques e distribuidos que inclui o armazenamento de água no solo, acoplado com uma análise clássica da estabilidade talude infinito em condições saturadas. Em este artigo avalia-se a influência da precipitação antecedente e condutividade hidráulica dos deslizamentos detonados por chuvas utilizando o modelo SHIA_Landslide. Os resultados obtidos neste estudo são semelhantes aos obtidos por diferentes autores na literatura. Os solos com altos valores de condutividade só precisam de chuvas curtas e intensas para falhar, e as condições de chuva antecedentes não desempenham um papel importante para estes tipos de solos.

PALAVRAS-CHAVE: Modelos físicos; Deslizamentos detonados pelas chuvas; SHIA_Landslide.

\section{INTRODUCCIÓN}

Aunque los movimientos en masa hacen parte del ciclo geomorfológico continuo y natural que modela la superficie terrestre, también son una de las principales causas de pérdidas humanas y económicas alrededor del mundo, especialmente en ambientes tropicales y zonas de montaña, por la expan- sión y asentamiento urbano en áreas susceptibles (Schuster, 1996; Sidle y Ochiai, 2006). Es por esta razón que la evaluación de la amenaza y la capacidad de predecir estos fenómenos han sido temas de gran interés para la comunidad científica en la implementación de sistemas de alerta temprana (Caine, 1980; Montgomery y Dietrich, 1994; Finlay et al., 1997; Crosta, 1998; Terlien, 1998; Crozier, 1999; 
Polemio y Petrucci, 2000; Iverson, 2000; NOAAUSGS, 2005; Restrepo et al., 2008; Larsen, 2008).

Los deslizamientos son causados por una gran variedad de factores que exigen adoptar un enfoque interdisciplinario, integrando conceptos de la geomorfología, hidrología y mecánica de suelos (Crosta y Frattini, 2008). Sin embargo, solo un único factor externo se convierte en el factor detonante que desencadena un movimiento en masa. En ambientes tropicales y terrenos montañosos como Los Andes colombianos, un alto porcentaje de estos movimientos en masa son provocados por intensas lluvias o eventos de larga duración (Aristizábal y Gómez, 2007), y se caracterizan por su pequeño espesor $(0,3-2 \mathrm{~m})$, superficie de falla plana paralela a la superficie del terreno, y escarpe con área pequeña (Anderson y Sitar, 1995). Estos movimientos se generan como consecuencia de eventos de lluvia por el rápido aumento en la presión de poros positiva o por la pérdida de componente de cohesión aparente (Wang y Sassa, 2003; Terlien, 1998; Crosta, 1998; Crosta y Frattini, 2003).

Collins y Znidarcic (2004) proponen dos mecanismos de falla diferentes para deslizamientos detonados por lluvias. En el primer mecanismo la falla se produce debido al aumento de la presión de poros positiva, en donde posterior a la falla del terreno el material desplazado, por medio de procesos de licuefacción estática y la reducción rápida de la resistencia a la cizalladura en condiciones no drenadas, se convierte en un flujo que se propaga ladera abajo, aumentando el volumen inicial del material por arranque y transporte a lo largo de la superficie de desplazamiento (Wang y Sassa, 2003; Wieczorek y Guzzetti, 2000). Algunos autores señalan la iniciación del movimiento y su movilización como simultáneos y no diferenciables (Eckersley, 1990; Anderson y Sitar, 1995). El segundo mecanismo de falla se produce bajo presiones de poros negativas donde el material está todavía en un estado no saturado; la falla ocurre debido a la reducción de la succión, y la masa que falla se comporta como un cuerpo rígido. (ver Figura 1).
Figura 1. Posibles mecanismos de deslizamientos detonados por lluvias: en la 1A por la formación y aumento del nivel freático colgado; $y$ en la 1B por avance de un frente húmedo a partir de la superficie del terreno. ß: pendiente, b: ancho de celda, Z: profundidad de superficie de falla, $Z_{w}$ : profundidad de saturación, $\mathrm{\gamma}$ : peso unitario del suelo, U: presión de poros, P: componente normal del peso, T: componente tangencial del peso.

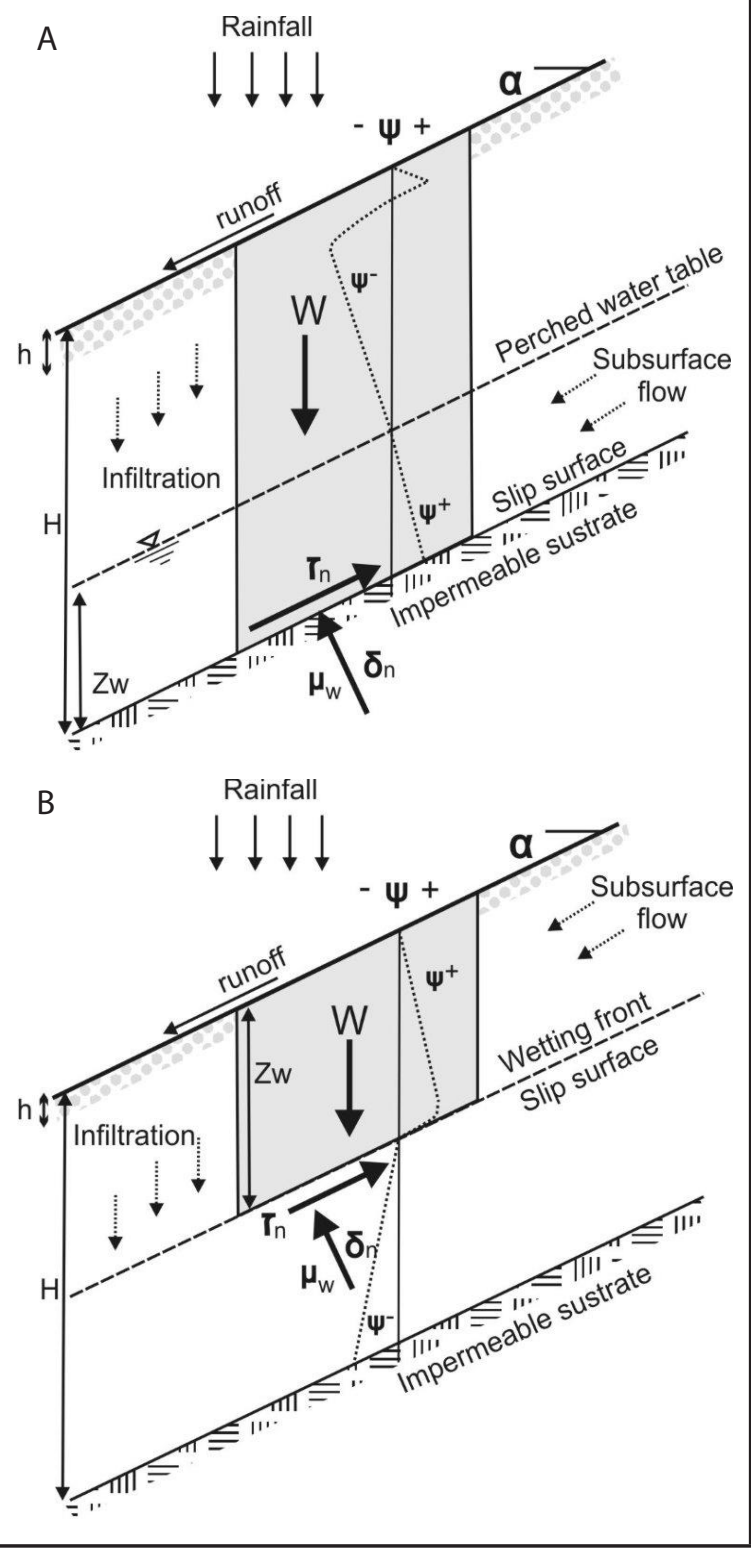

Recientemente, Aristizabal et al. (2016) acoplaron un modelo conceptual hidrológico con un modelo geotécnico de base física, al cual denominaron 
SHIA_Landslide (Simulación HIdrológica Abierta para Deslizamientos detonados por lluvias). SHIA_Landslide es una contribución original y significativa que ofrece una nueva perspectiva de análisis de los procesos de deslizamientos superficiales a escala de cuenca, mediante la incorporación de un modelo hidrológico de tanques completo y distribuido, que incluye el almacenamiento de agua en el suelo, junto con un análisis geotécnico y clásico de talud infinito bajo condiciones de saturación. SHIA_Landslide es accesible a través de una interfaz gráfica de usuario (GUI) que permite a este interactuar con el modelo.

Uno de los alcances del modelo SHIA_Landslide es mejorar la comprensión del mecanismo asociado con la inestabilidad de las laderas y la infiltración de la precipitación en las zonas montañosas situadas en ambientes tropicales, donde la presión demográfica está llevando a la expansión del desarrollo en áreas propensas a deslizamientos.

El presente trabajo evalúa la influencia de la conductividad hidráulica y lluvia antecedente en la ocurrencia de movimientos en masa detonados por lluvia utilizando modelación con base física a través del modelo SHIA_Landslide. Para lo cual se utilizó el evento de septiembre 21 de 1990 en la quebrada La Arenosa donde un evento de lluvia intenso detonó 823 deslizamientos superficiales.

\section{MECANISMO DE LOS}

\section{DESLIZAMIENTOS DETONADOS POR LLUVIAS EN AMBIENTES TROPICALES}

Los terrenos montañosos en ambientes tropicales se caracterizan por altos valores de pluviosidad y profundos perfiles de meteorización en condiciones saturadas o humedades cercanas a condiciones de campo. Es por esta razón que los deslizamientos superficiales detonados por lluvias en ambientes tropicales están controlados por el perfil de meteorización y la capacidad de almacenamiento del agua en el suelo.
De acuerdo con los estudios realizados por Little (1969), Deere y Patton (1971) y Anon (1981), el perfil del suelo residual tropical se puede simplificar en tres horizontes básicos con características de ingeniería específicas y particulares. Inicialmente un horizonte denominado suelo residual, donde el material corresponde completamente a suelo, y no se identifican estructuras de la roca parental. A lo largo de este horizonte se dan cambios mineralógicos significativos que afectan el volumen del suelo y aumentan la permeabilidad y conductividad hidráulica. Este horizonte superior del suelo está influenciado por la zona de raíces de la vegetación, y procesos de origen animal que generan estructuras de macroporos y conductos. Estas estructuras desarrollan un sistema de porosidad secundaria, aumentando sustancialmente la conductividad hidráulica de los suelos que actúan como trayectorias de flujos preferenciales, originando durante eventos de lluvia un rápido aumento del grado de saturación y de la presión de poros. Por debajo del suelo residual se encuentran horizontes saprolíticos, los cuales corresponden también a material tipo suelo, pero las estructuras originales de la roca parental se conservan. Los cambios mineralógicos y de volumen, así como la influencia de la superficie terrestre, no son importantes en estos horizontes, dando como resultado una reducción en la conductividad hidráulica en varios órdenes de magnitud, y un marcado contraste con respecto al suelo residual. Por último, están los horizontes con comportamiento y características similares a la roca y de muy baja conductividad (ver Figura 2).

En términos del contenido de agua a lo largo del perfil de meteorización es importante destacar que la capacidad de almacenamiento es función de los poros disponibles en el suelo y del grado de saturación. Del agua que se precipita sobre la superficie del terreno, una parte importante se infiltra en el suelo, dando inicio al llenado de los poros vacíos entre las partículas del suelo. Este, en condiciones no saturadas, tiene la capacidad de mantener el agua dentro de sus poros más pequeños debido a fuerzas 
de tensión (Fredlund y Rahardjo, 1993). Este contenido de agua se conoce como agua capilar. Si la gravedad ejerce una fuerza suficiente para superar la tensión superficial, el exceso de agua percola a los horizontes inferiores del perfil de meteorización. Este contenido de agua se conoce como agua gravitacional, y este umbral, en términos de contenido de agua, se denomina capacidad de campo (Wfc). La capacidad de campo se alcanza cuando la succión del suelo es de aproximadamente $30 \mathrm{kPa}$ en arcilla o 10 $\mathrm{kPa}$ en suelos arenosos (Richards y Weaver, 1944; Saxton y Rawls, 2006). El exceso de agua por encima de la capacidad de campo drena libremente, de acuerdo con la conductividad hidráulica del suelo, hasta alcanzar de nuevo la capacidad de campo. El contenido de agua por debajo del punto de capacidad de campo se elimina por evapotranspiración hasta que se alcanza el punto de marchitez permanente (Wpwp). En este punto, el contenido volumétrico de agua es demasiado bajo para que la planta pueda extraer agua del suelo, lo que corresponde aproximadamente a $1.500 \mathrm{kPa}$ de succión (Veihmeyer y Hendrickson, 1928; Saxton y Rawls, 2006).

Figura 2. Perfil general de meteorización de suelos en ambientes tropicales

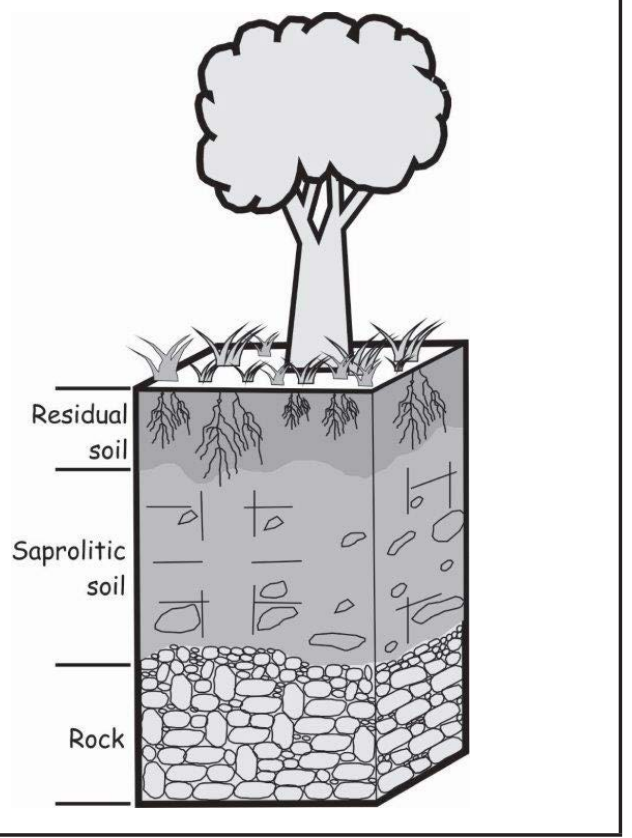

Bajo dicho esquema del perfil de meteorización y comportamiento del agua, el contraste existente entre suelo residual y suelo saprolítico, en términos de conductividad hidráulica, da lugar a que gran parte del agua gravitacional que percola a lo largo del suelo residual no pueda drenar hacia niveles más profundos como el suelo saprolítico, y se forme un nivel freático colgado. Este proceso de saturación del perfil de meteorización de abajo hacia arriba genera un flujo subsuperficial paralelo a la superficie del terreno. En términos de estabilidad de laderas esto significa que la superficie potencial de falla se encuentra a lo largo del contacto entre el suelo residual relativamente permeable y el suelo saprolítico, relativamente impermeable. Cuando se alcanza una altura crítica del nivel freático colgado, y por consiguiente el aumento de presiones de poros positiva, se producen movimientos en masa de tipo combinados, de acuerdo con la clasificación de Cruden y Varnes (1996). Donde inicialmente corresponden a deslizamientos planares que rápidamente se convierten en flujos por fenómenos de licuefacción estática, debido a la rápida reducción de la resistencia al cortante en condiciones no drenadas (Anderson y Sitar, 1995).

Esto significa que las condiciones no saturadas del suelo dominan al inicio de los eventos de lluvia, o cuando la lluvia antecedente ha sido muy baja, y solo durante estos momentos los deslizamientos pueden ocurrir por reducción de la succión. Sin embargo, después de un corto período de lluvia, las condiciones saturadas prevalecen y controlan la ocurrencia de deslizamientos superficiales.

\section{MODELO SHIA_LANDSLIDE}

SHIA_Landslide es un programa en FORTRAN para evaluar la estabilidad de las laderas en una cuenca ante condiciones de lluvia, acoplando un modelo hidrológico conceptual y distribuido con un modelo geotécnico de equilibrio límite utilizando el criterio de falla de Mohr-Coulomb.

El modelo requiere un evento o serie de lluvia como entrada, lo que provoca un aumento del nivel 
freático colgado y, en consecuencia, un aumento de las presiones de poros positivas que conduce a la reducción de las condiciones de estabilidad de las laderas. Una descripción más detallada de la SHIA_ Landslide se puede encontrar en Aristizábal (2014) y Aristizábal et al. (2015).

Para modelar los procesos hidrológicos a nivel de cuenca y ladera, el modelo SHIA_Landslide utiliza la metodología de Simulación Hidrológica Abierta y Distribuida (SHIA) desarrollada por Vélez (2001), la cual considera diferentes enfoques lineales o no lineales para estimar los flujos de agua en la dirección vertical y horizontal.

El modelo representa la cuenca como un conjunto de celdas interconectadas siguiendo la configuración topográfica, tomada de un Modelo de Elevación Digital. Cada celda de la cuadrícula corresponde a un sistema de cinco tanques interconectados que se comunican con su respectivo tanque en la celda, aguas abajo. Los primeros cuatro tanques representan los procesos de producción de escorrentía de la cuenca, mientras que el último tanque representa el flujo de agua a través de la red fluvial. De este modo las variables de estado del modelo son los contenidos de agua en cada tanque durante cada paso de tiempo de la simulación, y los flujos entre tanques representan los procesos físicos de intercambio de agua que son función del esquema conceptual adoptado, el tipo de tanque y las características morfológicas e hidrológicas del suelo presente en cada celda.

El primer tanque de almacenamiento, llamado almacenamiento capilar o estático, representa la intercepción y detención de agua en charcos y el almacenamiento del agua capilar en el suelo. El parámetro del suelo fundamental en este tanque es la capacidad máxima de almacenamiento de agua del suelo, o "agua útil" en el suelo, el cual es una entrada al modelo función de la capacidad de campo y profundidad de raíces. La única salida de este almacenamiento es la evapotranspiración real.

El segundo tanque se denomina el almacenamiento superficial y representa el agua que no se ha infiltrado y forma la escorrentía superficial. Puesto que ya se ha derivado previamente cierta cantidad de agua para el almacenamiento estático del suelo, se considera que la capacidad de infiltración se puede asociar a la conductividad hidráulica saturada del suelo, la cual es un parámetro de entrada al modelo. Para la escorrentía superficial se estima la velocidad de flujo función de la magnitud del flujo través de la ecuación de Manning:

$$
V_{2}=\frac{\xi A^{(2 / 3) e_{1}} \beta^{1 / 2}}{\mathrm{n}}
$$

donde $\mathrm{A}$ es el área de la sección de flujo, $\beta$ la pendiente de la ladera, $\mathrm{n}$ el coeficiente de Manning, y $\xi$ y $e_{1}$ son parámetros asociados con el tipo de superficie. Para flujos sobre terrenos naturales, Parsons et al. (1994) recomiendan valores de 0,038 y 0,315 , respectivamente.

El tercer tanque representa el almacenamiento de agua gravitacional contenida en el suelo residual entre la capacidad de campo y la saturación, lo cual corresponde al nivel freático colgado responsable de la reducción de las condiciones de estabilidad de la ladera y que se puede mover al tanque inferior por percolación o hacer parte del flujo subsuperficial. Los dos flujos se relacionan con la conductividad hidráulica saturada del sustrato rocoso, parámetro de entrada al modelo.

El cuarto tanque corresponde al almacenamiento subterráneo, donde el flujo de agua vertical representa la salida del sistema y el flujo horizontal representa el flujo base. Finalmente el quinto tanque representa los cauces de la red de drenaje.

Para la translación del flujo a lo largo de la red de drenajes se utiliza la metodología propuesta por Vélez (2001), denominada la "Onda Cinemática Geomorfológica" (OCG). La OCG es una simplificación de las ecuaciones de Saint Venant, en la que se desprecian los términos inerciales y de presión. El GKW, utilizando el cálculo de la rugosidad, correlaciona las ecuaciones propuestas por Leopold y Maddock (1953), que relacionan la geometría de la sección de flujo y la velocidad en la sección con el caudal utilizando ecuaciones potenciales, con la ecuación de 
Manning, que relaciona la velocidad de flujo y la sección transversal de flujo. Estas correlaciones permiten obtener una ecuación para la velocidad del flujo en el canal como una función de la geometría de canal y la geomorfología del terreno:

$$
V_{5}=\left[\frac{A\left(\frac{2}{3}-\varepsilon \theta\right)\left(1-\alpha_{2}\right) \beta\left(\frac{1}{2}-\varepsilon \theta\right)}{c_{n} c_{d}^{\varepsilon}\left(c_{1} k_{1}^{\left(\alpha_{1}-\alpha_{2}\right)} \Lambda^{\varphi\left(\alpha_{1}-\alpha_{2}\right)}\right)^{(2 / 3-\varepsilon \theta)}}\right]^{\frac{1}{1+\alpha_{2}\left(2 / 3^{-\varepsilon \theta)}\right.}}
$$

La OCG requiere nueve exponentes y coeficientes independientes, que pueden ser obtenidos de un estudio regional geomorfológico de zonas hidrológicamente homogéneas; para esto, han sido realizados estudios empíricos por varios autores que proponen diferentes valores de acuerdo con las condiciones locales (Frances et al., 2012). La Tabla 1 presenta los valores de los parámetros regionales constantes propuestos por Vélez (2001) y Frances et al. (2007).

\begin{tabular}{|c|c|c|}
\hline $\begin{array}{c}\text { Parámetro de la } \\
\text { OCG }\end{array}$ & Rango & Adoptado \\
\hline $\mathrm{K}_{1}$ & $0,5-0,75$ & 0,6 \\
\hline \multirow[t]{8}{*}{$\varphi$} & $0,65-0,8$ & 0,75 \\
\hline & $0,5-5,75$ & 3,26 \\
\hline & $0,34-0,55$ & 0,5 \\
\hline & $0,05-0,2$ & 0,2 \\
\hline & $0,5-50,0$ & 20 \\
\hline & $0,5-2.75$ & 1,26 \\
\hline & $0,025-0,07$ & 0,047 \\
\hline & $0,125-0,18$ & 0,1667 \\
\hline
\end{tabular}

\section{Figura 3. Modelo SHIA_Landslide}

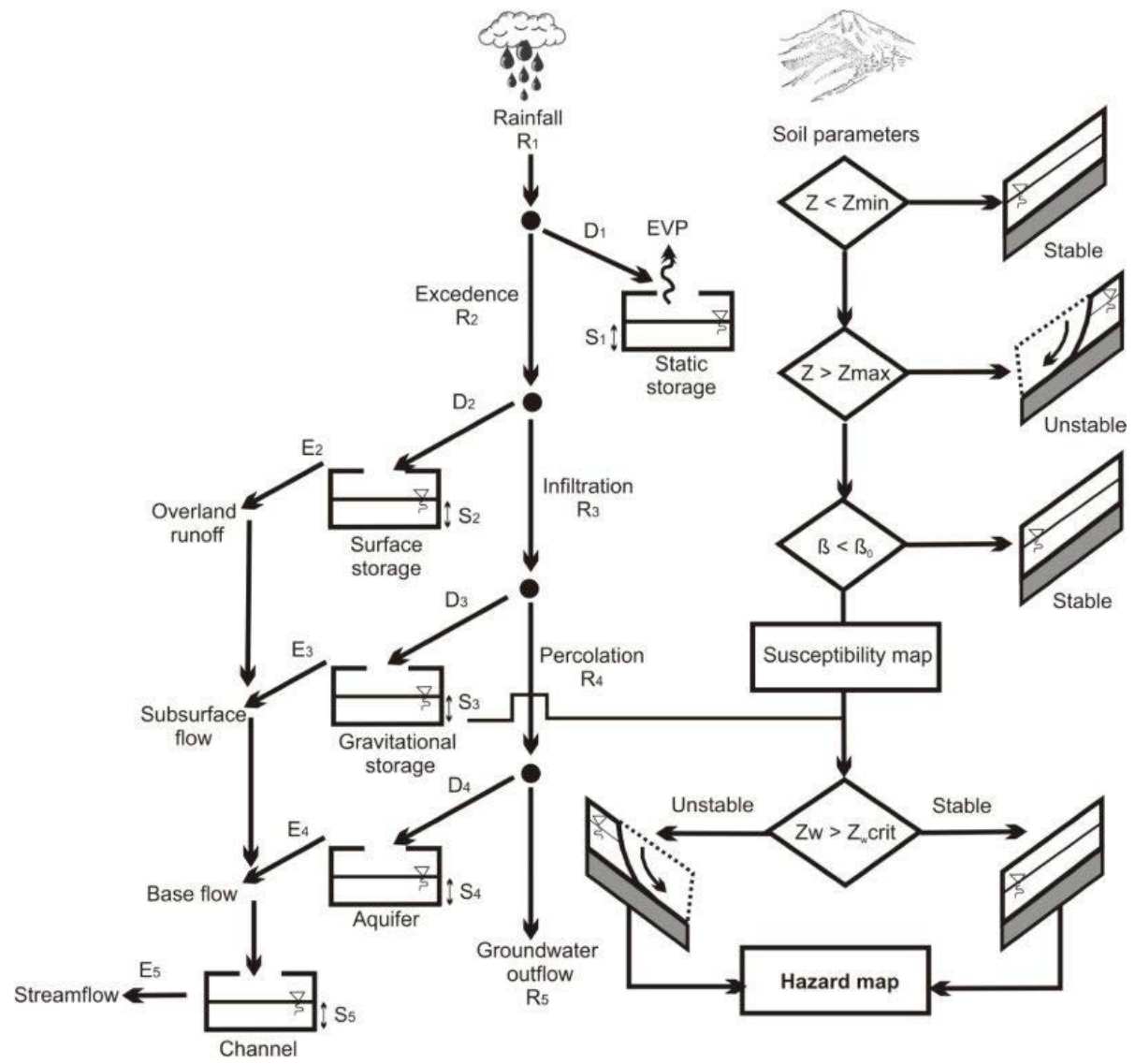


Para evaluar la estabilidad de taludes después de la simulación hidrológica de la cuenca y ladera, SHIA_Landslide utiliza un análisis de estabilidad basado en la física del modelo conocido como "talud infinito". Según Graham (1984), laderas con un flujo subsuperficial constante y paralelo a la ladera, con un nivel de agua por encima de la superficie de falla, el factor de la seguridad es:

$$
F S=\frac{C^{\prime}+\left(\gamma Z-\gamma_{w} Z_{w}\right) \operatorname{Cos}^{2} \beta \operatorname{Tan} \phi}{\gamma Z \operatorname{Sin} \beta \operatorname{Cos} \beta}
$$

donde C' es la cohesión efectiva, $\gamma$ es peso unitario del suelo, $Z$ es el espesor del suelo medido verticalmente, $\gamma_{w}$ es peso unitario del agua, $\beta$ es la pendiente de la ladera, y $\varphi$ es el ángulo de fricción.

Para el proceso de calibración, SHIA_Landslide incluye el modelo propuesto por Frances et al. (2007). Los parámetros en cada celda se dividen en dos componentes, una característica hidrológica o geotécnica y un factor de corrección común a todas las células que considera los errores de modelación. Los factores de corrección representan los efectos de escala espaciales y temporales, al igual que los errores del modelo y los parámetros de entrada, dejando a las características hidrológicas o geotécnicas libres de estos problemas, manteniendo el significado físico del parámetro (Frances et al., 2012).

Para evaluar el desempeño del modelo, y en caso que se cuente con datos observados de caudal en cualquier punto de la cuenca, SHIA_Landslide genera como valor de salida el error cuadrático medio (RMSE) y el coeficiente de eficiencia de Nash-Sutcliffe (NS). Por otra parte, el modelo utiliza la precipitación total sobre la zona de captación y las salidas de la cuenca para realizar un balance de agua, considerando el almacenamiento de agua en la cuenca.

En términos de resultados, el modelo genera cuatro productos de salida para cada simulación. El hidrograma y la variación del nivel freático colgado para cualquier punto de la cuenca, el mapa de susceptibilidad por deslizamientos de la cuenca, y un mapa de amenaza por deslizamientos, que corres- ponde a la distribución espacial de los deslizamientos provocados por el evento de lluvia simulado.

La Figura 3 presenta la estructura de diagrama de flujo del modelo acoplado, SHIA_Landslide.

\section{IMPLEMENTACIÓN DEL MODELO}

\section{SHIA_LANDSLIDE}

Para evaluar la influencia de la lluvia antecedente y la conductividad hidráulica del suelo en la ocurrencia de deslizamientos superficiales detonados por lluvias, se implementó el modelo SHIA_ Landslide en la cuenca de la quebrada La Arenosa, típica de ambientes de montañas y zonas tropicales. La cuenca del río La Arenosa tiene un área de 9,91 km² y está localizada al suroriente de la Cordillera Central en el departamento de Antioquia. La zona tiene un clima tropical húmedo con una media precipitación anual de $4.300 \mathrm{~mm}$ y una temperatura media anual de $23^{\circ}$ (IGAC 2007). La geología se compone de suelos residuales de rocas granodioriticas cubiertos en áreas de pendiente suave con depósitos fluviotorrenciales (Mejía y Velásquez, 1991).

El 21 de septiembre de 1990, un evento de lluvia de alta intensidad afectó a la cuenca La Arenosa. En menos de 3 horas, $208 \mathrm{~mm}$ se precipitaron sobre la cuenca, lo que provocó 823 movimientos en masa. Durante este evento, la población se vio fuertemente afectada, 20 personas murieron y 260 tuvieron que ser evacuadas, 27 casas fueron destruidas y otras 30 fueron afectadas, varios puentes y más de $100 \mathrm{~m}$ de la carretera quedaron en ruinas. La central de energía hidroeléctrica Calderas, propiedad de ISAGEN S.A. E.S.P., se inundó y se vio severamente afectada por grandes bloques transportados por la quebrada La Arenosa. Las pérdidas totales se estimaron en más de US \$ 6 millones (Hermelin et al., 1992).

Un análisis posterior al evento con fotos aéreas e investigaciones de campo permitió una reconstrucción parcial del patrón y las características de los deslizamientos en la cuenca de La Arenosa. INTEGRAL (1990) y Mejía y Velásquez (1991) realizaron un inventario detallado de los deslizamientos provocados 
durante el evento y una descripción completa. Mejía y Velásquez (1991) reportaron 699 movimientos en la cuenca de La Arenosa.

\begin{tabular}{|c|c|c|}
\hline PARÁMETRO & $\begin{array}{l}\text { Alluvioto- } \\
\text { rrencial }\end{array}$ & $\begin{array}{c}\text { Suelos } \\
\text { de rocas } \\
\text { greaníticas }\end{array}$ \\
\hline Pendiente $\left({ }^{\circ}\right)$ & $0-5$ & $0-62$ \\
\hline $\begin{array}{l}\text { Capacidad máxima } \\
\text { de almacenamiento } \\
\text { estático del agua }(\mathrm{mm})\end{array}$ & $63-135$ & $63-135$ \\
\hline $\begin{array}{l}\text { Capacidad máxima de } \\
\text { almacenamiento de } \\
\text { agua gravitacional }(\mathrm{mm})\end{array}$ & $191-465$ & $191-465$ \\
\hline $\begin{array}{l}\text { Conductividad } \\
\text { hidráulica saturada del } \\
\text { suelo residual }(\mathrm{cm} / \mathrm{h})\end{array}$ & 0.479 & 1.96 \\
\hline $\begin{array}{l}\text { Conductividad } \\
\text { hidráulica saturada del } \\
\text { suelo saprolítico }(\mathrm{cm} / \mathrm{h})\end{array}$ & 0,0799 & 0,0799 \\
\hline $\begin{array}{l}\text { Conductividad } \\
\text { hidráulica saturada de la } \\
\text { roca }(\mathrm{cm} / \mathrm{h})\end{array}$ & $8 \times 10^{-8}$ & $8 \times 10^{-8}$ \\
\hline Cohesión (kPa) & 1 & 5 \\
\hline Ángulo de fricción ( ${ }^{\circ}$ ) & 34 & 24 \\
\hline $\begin{array}{l}\text { 'Peso unitario del suelo } \\
\text { saturado }\left(\mathrm{kN} / \mathrm{m}^{3}\right)\end{array}$ & 20 & 18 \\
\hline $\begin{array}{l}\text { Espesor del suelo } \\
\text { residual }(\mathrm{m})\end{array}$ & $2,5-2,8$ & $1,2-2,8$ \\
\hline
\end{tabular}

Para la implementación del modelo SHIA_ Landslide se requieren como parámetros de entrada tres mapas morfométricos: área acumulada, dirección del flujo, y pendiente; y nueve mapas de las propiedades hidrológicas y geomecánicas del suelo: espesor, cohesión, peso saturado, ángulo de fricción, conductividad hidráulica saturada del suelo residual, conductividad hidráulica saturada del suelo saprolítico, conductividad hidráulica saturada de la roca, capacidad máxima de almacenamiento estático del agua, y la capacidad máxima de alma- cenamiento de agua gravitacional. Los parámetros morfométricos se calcularon utilizando ArcGIS 10.1 herramientas hidrológicas y un DTM proporcionada por el Instituto Geográfico Agustín Codazzi (IGAC) con un tamaño de trama de $10 \mathrm{~m}$. Y los parámetros de suelo se obtuvieron del mapa de suelos elaborado por el IGAC (2007), y las descripciones de suelos, pruebas de campo y análisis de laboratorio de muestras de suelo realizadas por Mejía y Velásquez (1991) e INTEGRAL (1990) (Tabla 2).

Series de precipitación con resolución horaria entre los años 2007 y 2012 de estaciones de la cuenca La Arenosa fueron utilizadas para la calibración y validación del componente hidrológico del modelo. Y para el componente geotécnico se utilizó el evento de septiembre 21 de 1990, del cual se contaba un inventario detallado de los deslizamientos. El proceso de calibración y validación del modelo puede consultarse en detalle en Aristizábal (2014) y Aristizábal et al. (2015).

\section{RESULTADOS Y DISCUSIÓN}

Aunque la lluvia antecedente como un factor condicionante en la ocurrencia de deslizamientos es ampliamente discutida en la literatura, su influencia es difícil de cuantificar, ya que depende de varios factores, entre ellos la conductividad hidráulica y humedad del suelo. La conductividad hidráulica es una propiedad básica del suelo, e indudablemente juega un papel esencial desde el punto de vista del balance que debe existir entre la generación de la presión de poros y su disipación, lo cual es fundamental para la inicialización del movimiento (Wang y Shibata, 2007).

Para evaluar el efecto de la lluvia antecedente en la cuenca de la quebrada La Arenosa se evaluó la respuesta de la cuenca para el evento del 21 de septiembre de 1990, modificando las condiciones de lluvia de los tres meses y 24 horas antecedentes, y posteriormente evaluando los cambios presentados en el nivel freático colgado de algunas celdas seleccionadas dentro de la cuenca. 
Figura 4. Respuesta de una celda con área aferente de $800 \mathrm{~m}^{2}$ antes modificaciones en los valores de conductividad hidráulica (Ks).

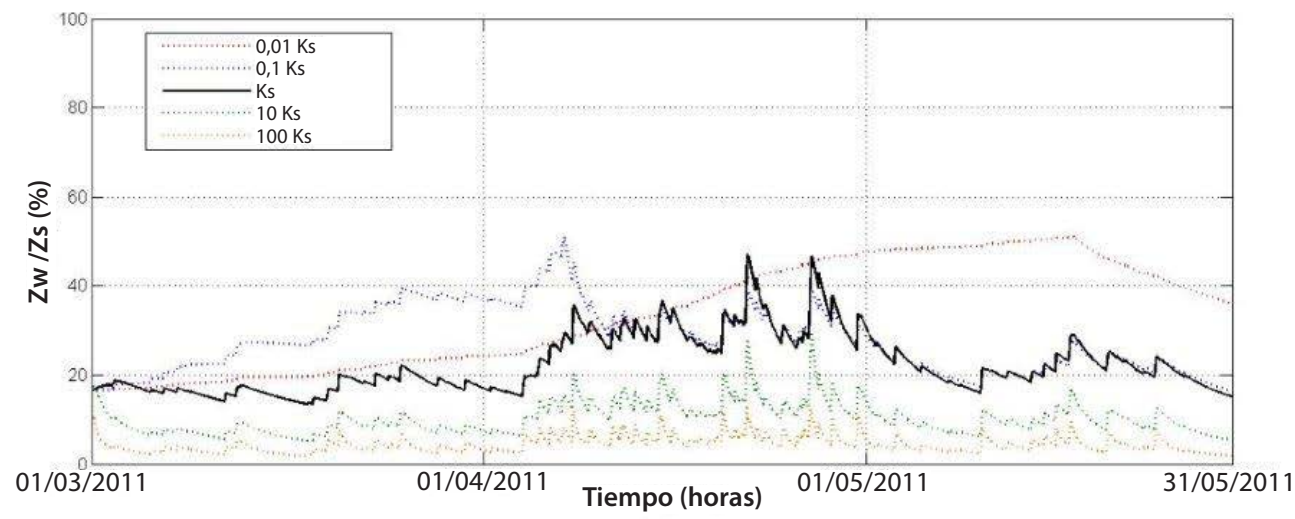

De acuerdo con los resultados obtenidos, el incremento de la lluvia antecedente en los tres meses previos al evento de 21 de septiembre 1990 no generó ningún cambio significativo en el pico del nivel freático colgado para las celdas evaluadas. De igual forma, el incremento de la lluvia antecedente en las 24 horas previas al evento tampoco representó cambios en el nivel freático.

Del mismo modo, se estudió no solo la influencia del volumen de agua precipitada sino también la posible influencia del hietograma de diferentes tormentas. Cuatro patrones representativos de tormentas a escala horaria fueron utilizados para un periodo de tiempo de 1000 horas y una intensidad máxima de lluvia de $50 \mathrm{~mm} / \mathrm{h}$. El patrón denominado uniforme conserva la intensidad de la lluvia igual (50 mm/h) durante toda la simulación. El patrón avanzado inicia con la intensidad máxima y disminuye progresivamente hasta una intensidad de 0 $\mathrm{mm} / \mathrm{h}$. El patrón denominado retroceso inicia con una intensidad de lluvia de $0 \mathrm{~mm} / \mathrm{h}$, aumentando progresivamente hasta $50 \mathrm{~mm} / \mathrm{h}$. Finalmente, el patrón central comienza con una intensidad de lluvia de $0 \mathrm{~mm} / \mathrm{h}$, alcanzando la intensidad máxima a la mitad del periodo período de simulación y disminuye progresivamente hasta alcanzar una intensidad de lluvia igual a la inicial de $0 \mathrm{~mm} / \mathrm{h}$. Los cuatro patrones de lluvia produjeron el mismo número de celdas inestables. No se observaron diferencias para la cuenca de La Arenosa si el pico máximo de intensidad de la tormenta es al principio, en el medio o al final. La diferencia está en el momento en el cual ocurren los deslizamientos; debido al hecho que la mayoría de los deslizamientos se producen durante el pico de la tormenta.

Para evaluar la sensibilidad del modelo a la conductividad hidráulica en condiciones saturadas, se utilizó una serie de lluvia de tres meses de longitud y resolución horaria, y se evaluó el cambio en el nivel freático colgado ante variaciones en la cuenca de los valores de conductividad (ver Figura 4). Estas variaciones son hipotéticas como un experimento numérico mediante análisis de sensibilidad, y no responden a condiciones de la realidad, ya que no se consideran posibles cambios en características del suelo que pueden estar relacionadas.

Cuando la conductividad hidráulica es reducida en dos órdenes de magnitud, los picos de nivel freático colgado desaparecen, con un aumento constante que alcanza un pico máximo levemente superior al $50 \%$ del espesor del suelo. El nivel freático colgado mantiene sus niveles altos, incluso después de finalizar el evento de lluvia, lo que indica una menor capacidad para disipar las 
presiones de poros positivas del suelo debido a su baja conductividad.

Si la conductividad es reducida en un orden de magnitud, en términos generales se reducen los picos máximos, al principio se da un aumento constante del nivel freático, con pequeños picos que responden a eventos de lluvias cortas. Cuando alcanza una altura cercana al 50\% del espesor total del suelo para esta celda, hay un descenso brusco en la profundidad del nivel freático colgado, y el comportamiento cambia por completo, ajustándose al comportamiento de la conductividad del suelo, presentando a partir de este punto pequeños picos $\mathrm{y}$ valles que responden a eventos de lluvia sobre la cuenca. Para conductividades en uno y dos órdenes de magnitud mayores a la conductividad hidráulica del suelo se presentan pequeños incrementos del nivel freático, por debajo todos ellos del $30 \%$ del espesor del suelo, y con patrón similar a la conductividad medida.

Figura 5. Porcentaje de celdas potencialmente inestables que falla de acuerdo con el pico máximo de lluvia. En la $5 \mathrm{~A}$ la cuenca a escala con las coronas de los deslizamientos ocurridos en septiembre de 1990. En la 5B para un evento de Iluvia con picos de $20 \mathrm{~mm} / \mathrm{h}$ el $13 \%$ de las áreas potencialmente inestables fallan. En la 5C para un evento de lluvia con picos de $40 \mathrm{~mm} / \mathrm{h}$ el $32 \%$ de las áreas potencialmente inestables fallan. En la 5D para un evento de lluvia con picos de $80 \mathrm{~mm} / \mathrm{h}$ el $48 \%$ de las áreas potencialmente inestables fallan.

A

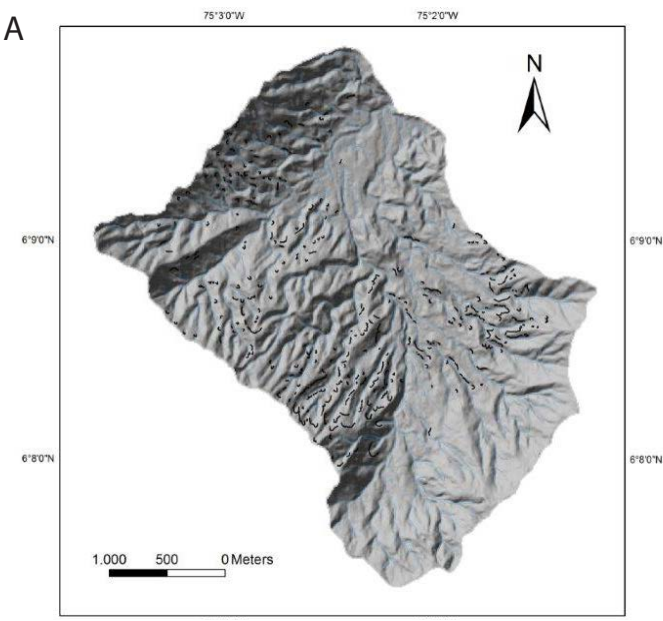

C

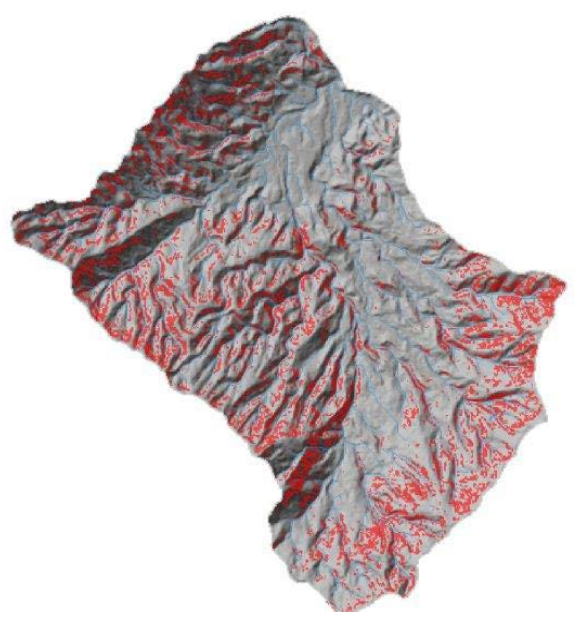

B

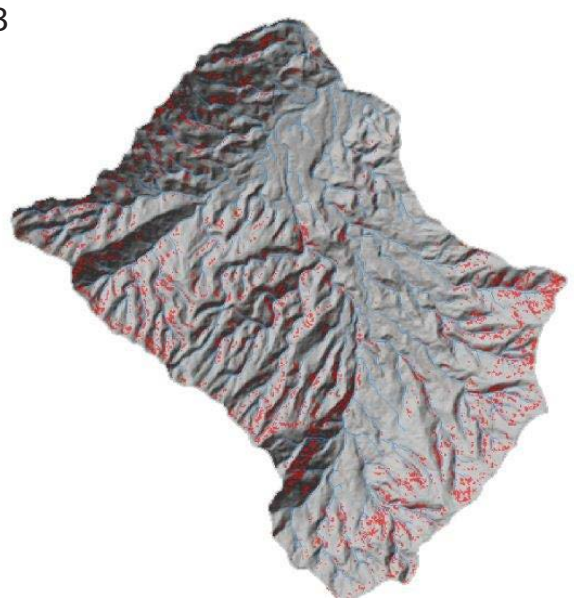

D

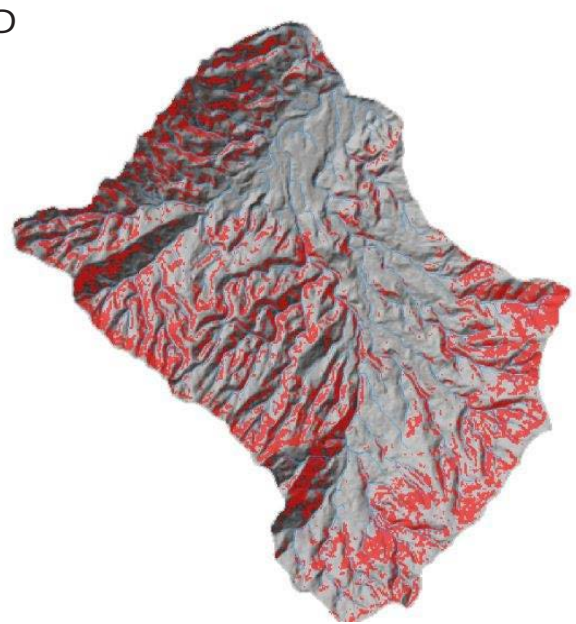


Estos resultados implican que los suelos arenosos que conforman la cuenca de la quebrada La Arenosa, caracterizados por su alta conductividad, la presión de poros positiva se reduce rápidamente después del evento de lluvia, por lo que requieren para su falla eventos de lluvia cortos e intensos, como el evento del 21 de setiembre de 1990. Y su capacidad de disipar presiones de poros por sus valores altos de conductividad limita que la lluvia antecedente tenga una influencia considerable dentro de su comportamiento.

Estos resultados son coherentes con los hallazgos de diferentes autores para suelos residuales con altas permeabilidades, característicos de suelos residuales arenosos de rocas graníticas. La lluvia antecedente y las condiciones de humedad no siempre juegan un papel determinante en los suelos (Rahardjo et al., 2008; Brand, 1985; Corominas y Moya, 1999). Inclusive algunos autores, tales como Tsaparas et al. (2002) y Rahardjo et al. (2007) registran diferencias en los mecanismos de falla asociados a la permeabilidad de los suelos, laderas con altas permeabilidades usualmente fallan por aumento de presiones de poros positiva, y laderas con bajas permeabilidades fallan por reducción de la succión. Por otra parte, Terlien (1998) encuentra que la diferencia en el número de días de lluvia antecedente depende de la profundidad de la superficie de falla, donde movimientos detonados por altas precipitaciones diarias son superficiales con profundidades menores a $2 \mathrm{~m}$, y movimientos detonados por lluvias antecedentes tienen superficies de falla profundas superiores a los $6 \mathrm{~m}$.

Como parte del análisis de sensibilidad de la conductividad hidráulica y lluvia antecedente, es posible, utilizando el modelo SHIA_Landslide, establecer umbrales de lluvia para predecir la ocurrencia de los deslizamientos. Para este caso se construyeron series de lluvia con picos máximos de lluvia de diferentes magnitudes y se evaluó el número de celdas que fallan.

Para una tormenta con picos de $20 \mathrm{~mm} / \mathrm{h}$, el $13 \%$ de las áreas potencialmente inestables fallan. Para picos de $40 \mathrm{~mm} / \mathrm{h}$, el 32\% de las áreas potencialmente inestables fallan. Y para un pico de 80 $\mathrm{mm} / \mathrm{h}, 48 \%$ de las áreas potencialmente inestables fallan (ver Figura 5).

Figura 6. Porcentaje de celdas potencialmente inestables de acuerdo con el pico máximo de lluvia en la cuenca de la quebrada La Arenosa

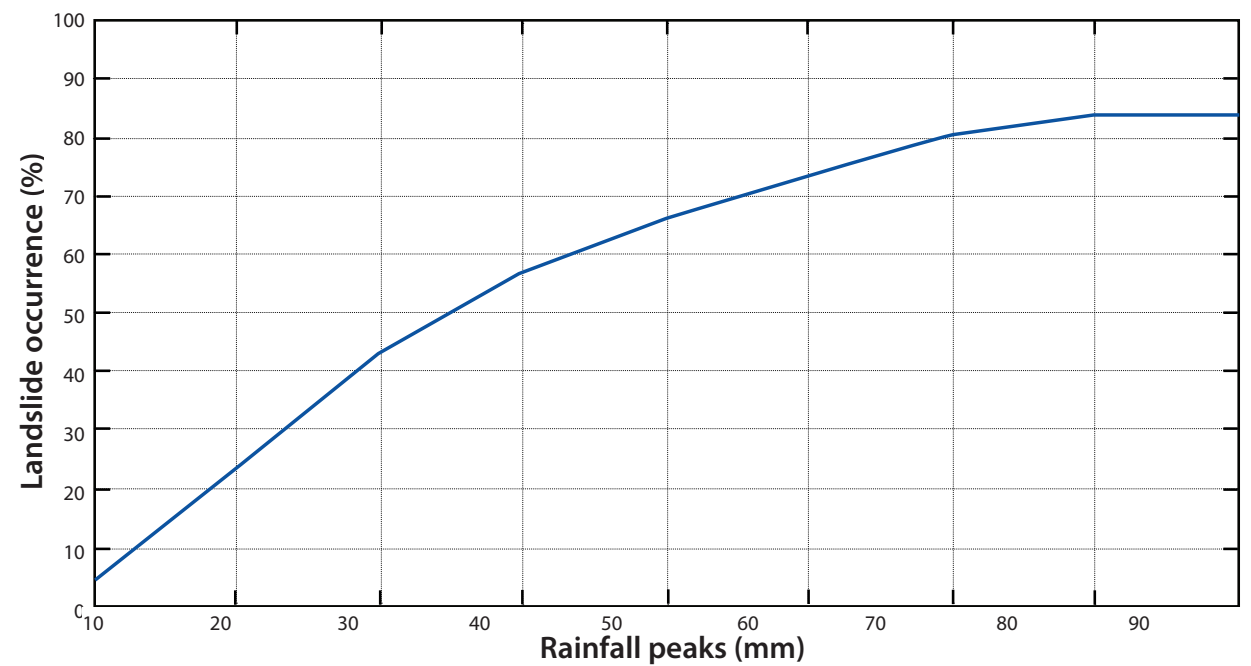


Figura 7. Porcentaje de celdas potencialmente inestables que fallan en función de la relación entre la altura del nivel freático colgado (Zw) y la profundidad del suelo residual (Zs)

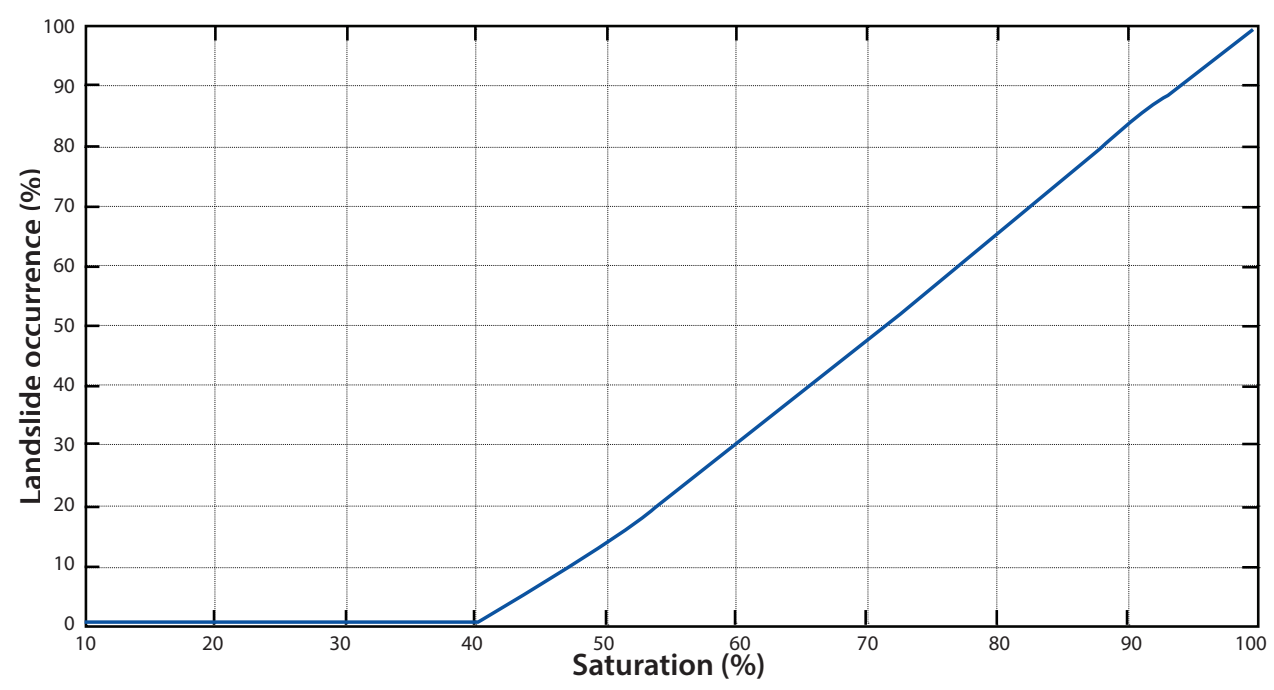

La Figura 6 presenta el porcentaje de celdas de la cuenca que fallan debido a la lluvia, con el porcentaje máximo obtenido ante diferentes picos de lluvia. El valor máximo obtenido está próximo al 50\% para picos cercanos a $100 \mathrm{~mm} / \mathrm{h}$. Para picos mayores no hay un aumento significativo de los deslizamientos. Este comportamiento señala una relación asintótica entre los picos de lluvias y la ocurrencia de deslizamientos. Existe una tendencia a aumentar linealmente para los pequeños picos de lluvias, y disminuye hasta llegar a un valor asintótico cercano al 50\%.

Estos resultados señalan que no existe un umbral mínimo de lluvia a partir del cual se presenta un marcado porcentaje mayor de deslizamientos; por el contrario, existe un umbral máximo cercano a $100 \mathrm{~mm} / \mathrm{h}$, por encima del cual no se presentan más deslizamientos sin importar la intensidad o duración de la lluvia. El valor del $50 \%$ de celdas que fallan significa un porcentaje máximo de ocurrencia de deslizamientos debido a la lluvia, el cual aparentemente emerge como un umbral máximo natural para la cuenca de la quebrada La Arenosa, asociada a sus condiciones hidrológicas y geotécnicas. Sin embargo, es necesario evaluar este comportamiento para otras cuencas, ya que dicho umbral podría también señalar una limitación del modelo impuesta por el proceso de calibración.

En este mismo sentido se evaluaron las condiciones de estabilidad de cada una de las celdas que conforman la cuenca ante el incremento en la altura del nivel freático colgado. Se simularon diferentes escenarios representando la altura del nivel freático colgado $(\mathrm{Zw})$ como un porcentaje de la profundidad del suelo que falla (Zs). El escenario donde $\mathrm{Zw}=\mathrm{Zs}$, corresponde al escenario más crítico, en el cual el nivel freático colgado para todas las celdas que conforman la cuenca coincide con la superficie del terreno. Bajo dicho escenario todas las celdas identificadas por el mapa de susceptibilidad como potencialmente inestables fallan. Para $\mathrm{Zw}=0,5 \mathrm{Zs}$, sólo el 13,6\% de las celdas potencialmente inestables fallan. Cuando el nivel freático colgado se acerca a condiciones de saturación, el número de células inestables aumenta considerablemente, cuando $\mathrm{Zw}$ $=0,8 Z$ s, el $65,6 \%$ de las celdas fallan.

La Figura 7 presenta el porcentaje de celdas de la cuenca inestables en función del aumento freático colgado para la cuenca de la quebrada La 
Arenosa. Cuando el nivel freático colgado aumenta por encima del $40 \%$ de la profundidad del suelo las áreas potencialmente inestables empiezan a fallar; por debajo de dicho valor no se presentan deslizamientos. Sin embargo, es importante señalar que dicho umbral no tiene un sentido físico a nivel de cuenca, ya que no es posible que todas las celdas simultáneamente presenten una relación o porcentaje Zw/Zs igual. Esta relación es función de cantidad de agua infiltrada, de las propiedades hidráulicas y geomecánicas del suelo, de la posición de la celda en la ladera y del espesor del suelo.

\section{CONCLUSIONES}

Los ambientes tropicales como Los Andes colombianos se caracterizan por perfiles de meteorización profundos y la presencia de lluvias durante gran parte del año. La mayoría de los deslizamientos que se presentan en estas regiones son inducidos por la lluvia. En Colombia, sin embargo, son pocos los eventos que se han caracterizado en detalle, que incluyan un inventario de deslizamientos. Para el evento de La Arenosa, el 21 de septiembre de 1990, Mejía y Velásquez (1991) e INTEGRAL (1990) llevaron a cabo una evaluación detallada y un inventario parcial, deslizamiento de tierra, lo que permite la implementación y evaluación de un modelo para el pronóstico de deslizamientos detonados por lluvia.

De acuerdo con los resultados obtenidos en la implementación del modelo SHIA_Landslide se puede establecer que el modelo es altamente sensible al espesor del suelo; la reducción en la profundidad del contacto entre suelo residual y saprolítico produce una mayor ocurrencia de deslizamientos. Con respecto a las propiedades geomecánicas existe una relación inversa esperada, donde aumentos en el ángulo de fricción o cohesión generan una reducción de las celdas que fallan. Y similar a resultados de numerosos modelos, el factor de seguridad es más sensible a los cambios en la cohesión, el espesor, y la pendiente, moderadamente sensible a los cambios en el ángulo de fricción, y poco sensible a los cambios en el peso unitario del suelo.
Los resultados encontrados en el presente estudio en términos generales señalan que laderas conformadas por suelos de alta conductividad requieren sólo eventos de lluvia cortos e intensos para su falla, y las condiciones de lluvia antecedente no juegan un papel importante.

\section{AGRADECIMIENTOS}

Los autores agradecen a la Fundación Wildsdorf Hans y la Asociación Colombiana de Geólogos y Geofísicos del Petróleo (ACGGP) por el apoyo financiero para la realización de esta investigación.

\section{REFERENCIAS}

Anderson, M.G.; Lloyd, D.M. (1991). Using a combined slope hydrology-stability model to develop cut slope design charts. Proc. Inst. Civ. Engineers, 91, 705-718.

Anderson, S.A.; Sitar N. (1995). Analysis of rainfall-induced debris flows. Journal of Geotechnical Engineering, pp. 544-552.

Aristizábal, E.; Vélez, J.I.; Martínez, H. (2016). SHIA_Landslide: a distributed conceptual and physically based model to forecast the temporal and spatial occurrence of shallow landslides triggered by rainfall in tropical and mountainous basins. Landslides,13, pp. 497-517.

Aristizábal, E. (2014). SHIA_Landslide: Developing a physically based model to predict shallow landslides triggered by rainfall in tropical environments. $\mathrm{Ph}$ Thesis, Universidad Nacional de Colombia, pp. 217.

Aristizábal, E.; Gómez, J. (2007). Inventario de emergencias y desastres en el Valle de Aburrá: originados por fenómenos naturales y antrópicos en el periodo 1880-2007. Revista Gestión y Ambiente, 10(2), pp. 17-30.

Brand, E.W. (1985). Predicting the performance of residual soil slopes. Proceedings 11th Int. Conf. Soil Mech. \& Found. Engineering. San Francisco, 5, pp. 2541-2578

Caine, N. (1980). The rainfall intensity - duration control of shallow landslides and debris flows. Geografiska Annaler, 62A(1-2), pp. 23-27.

Collins, B.D.; Znidarcic D. (2004). Stability analyses of rainfall induced landslides. Journal of Geotechnical and Geoenvironmental Engineering. $130(4)$, pp. 362-371. 
Corominas, J.; Moya, J. (1999). Reconstructing recent landslide activity in relation to rainfall in the Llobregat river basin, Eastern Pyrenees, Spain. Geomorphology, 30, 79-93.

Crosta, G. (1998). Regionalization of rainfall threshold: an aid for landslide susceptibility zonation. Enviromental Geology, 35(2-3), 131-145.

Crosta, G.; Frattini, P. (2003). Distributed modeling of shallow landslides triggered by intense rainfall. Natural Hazard and Earth System Sciences, 3, pp. 81-93.

Crosta, G.; Frattini, P. (2008). Rainfall-induced landslides and debris flows. Hydrological Processes, 22, pp. 473-477.

Cruden, D. M.; Varnes D.J. (1996). Landslide types and processes. En: A. K. Turner y R.L. Schuster (Editores): Landslides. Investigation and mitigation. Transportation Research Board Special Report 247. National Academy Press. Washington D.C. pp. 36-75.

Deere, D.U.; Patton F.D. (1971). Slope stability in residual soils. Proc., Fourth Pan American Conference on Soil Mechanics and Foundation Engineering, Puerto Rico. 1, pp. 87-170.

Eckersley, J.D. (1990). Instrumented laboratory flowslides. Geotechnique. London, England, 40(3), pp. 489502.

Finlay, P.J.; Fell, R.; Maguire, P.K. (1997). The relationship between the probability of landslide occurrence and rainfall. Can. Geotech. Journal, 34. pp. 811-824.

Frances, F.; Vélez, J.I.; Vélez, J.J. (2007). Split-parameter structure for the automatic calibration of distributed hydrological models. Journal of Hydrology, 332, pp. 226-240.

Frances, F.; Vélez, J.J.; Munera, J.C.; Medici, C.; Busii, G. (2012). Descripción del modelo conceptual distribuido de simulación hidrológica TETIS v.8. Universidad Politécnica de Valencia. 86 pp.

Fredlund, D.G.; Rahardjo, H. (1993). Soil mechanics for unsaturated soils, Wiley-Interscience, New York.

Graham, J. (1984). Methods of Stability Analysis, Department of Civil Engineering, University of Manitoba, Slope Instability: In D. Brunsden and D.B. Prior (Eds), John Wiley \& Sons Ltd., pp. 171-215.

Hermelin, M.; Mejía, O., Velásquez, E. (1992). Erosional and depositional features produced by a convulsive event, San Carlos, Colombia, September 21, 1990. Bulletin of the International Association of Engineering Geology, 45, pp. 89-95.
IGAC - Instituto Geográfico Agustín Codazzi (2007). Estudio general de suelos y zonificación de tierras del departamento de Antioquia. Bogotá. 207 p.

INTEGRAL S.A. (1990). Informe sobre daños en la central de calderas por la avalancha ocurrida en I quebrada LA Arenosa el 21 de septiembre de 1990 y su reparación. Internal Report Interconexión Eléctrica S.A. ISA.45 p.

Iverson, R. (2000). Landslide triggering by rain infiltration. Water Resources Research, 36(7), pp. 1897 1910.

Larsen, M.C. (2008). Rainfall-triggered landslides, anthropogenic hazards, and mitigation strategies. Advances in Geosciences, 14, pp. 147-153.

Leopold, L.B.; Maddock, T.J. (1953). Hydraulic geometry of stream channels and some physiographic implications. U. S. Geological Survey Professional Paper, 252, $55 \mathrm{p}$.

Little, A.L. (1969). The engineering classification of residual tropical soils. Proceedings of $7^{\text {th }}$ International Conference of Soil Mechanics and Foundation Engineering, 1, pp. 1-10.

Matula, M. (1981). Rock and soil description and classification for engineering geological mapping. Bulletin of International Association of Engineering Geology, 24, pp. 253-274.

Mejía, R.; Velásquez, M.E. (1991). Procesos y depósitos asociados al aguacero de septiembre 21 de 1990 en el Área de San Carlos (Antioquia). Tesis de grado, Universidad Nacional de Colombia, Medellín. 160 p.

Montgomery, D. R.; Dietrich, W. E. (1994). A physically based model for the topographic control on shallow landsliding. Water Resources Research, pp. 11531171.

Montgomery, D. R.; Sullivan, K.; Greenberg, M. (1998). Regional test of a model for shallow landsliding. $\mathrm{Hy}$ drol. Process.,12, pp. 943-955.

National Oceanic \& Atmospheric Administration NOAA, United Status Geological Survey USGS (2005). NOAA-USGS Debris flow warning system-final report, circular 1283. [Online] Disponible en: http.// pubs.usgs.gov/circ/2005/1283/.

Parsons, A.J.; Abrahams, A.D.; Wainwright, J. (1994). On determining resistance to interrill overland flow. Water Resources Research, 30(12), pp. 3515-3521.

Rahardjo, H.; Ong, T.H.; Rezaur, R.B.; Leong, E.C. (2007). Factors controlling instability of homogeneous soil slopes under rainfall. Journal of Geotechnical and 
Geoenvironmental Engineering, 133(12), pp. 15321543.

Rahardjo, H.; Leong, E.C.; Rezaur, R.B. (2008). Effect of antecedent rainfall on pore-water pressure distribution characteristics in residual soil slopes under tropical rainfall. Hydrological Processes, 22. pp. 506-523.

Restrepo, P.; Jorgensen, D.P.; Cannon, S.H.; Costa, J.E.; Laber, J.; Major, J.A.; Marter, B.; Purpura, J.; Werner, K. (2008). Prototype debris flow warning system for recently burned areas in Southern California. Bulletin of the Meteorological Society, Insights and Innovation, American Meteorological Society, pp. 1845-1851.

Richards, L.A.; Weaver, L.R. (1944). Moisture retention by some irrigated soils as related to soil moisture tension. Journal of Agricultural Research, 69, pp. 215-235.

Saxton, K.E.; Rawls, W.J. (2006). Soil Water Characteristic Estimates by Texture and Organic Matter for Hydrologic Solutions. Soil Science Society of America Journal, 70, pp. 1569-1578.

Schuster, R.L. (1996). Socioeconomic significance of landslides. In: A.K. Turner \& R.L. Schuster (Eds) Landslides Investigation and Mitigation. Transportation Research Board, National Research Council, Special Report 247, National Academy Press, Washington, DC, ISA. pp. 129-177.

Sidle, R.C.; Ochiai, H. (2006). Landslides: processes, prediction, and land use. Water Resources Monograph 18. American Geophysical Union, Washington D.C.

Tsaparas, I.; Rahardjo, H.; Toll, D.G.; Leong, E.C. (2002). Controlling parameters for rainfall-induced landslides. Computers and Geotechnics, 29, pp. 1-27.

Terlien, M.T.J. (1998). The determination of statistical and deterministic hydrological landslide-triggering thresholds. Environmental Geology, 35(2-3), pp. 124-130.

Veihmeyer, FJ.; Hendrickson, A.H. (1928). Soil moisture at permanent wilting of plants. Plant Physiol. 3(3), pp. 355-357.

Vélez, J.I. (2001). Desarrollo de un modelo hidrológico conceptual distribuido orientado a la simulación de crecidas. Valencia. Tesis Doctoral. Universidad Politécnica de Valencia.

Wang, G.; Sassa, K. (2003). Pore pressure generation and movement of rainfall-induced landslides: effects of grain size and fine particle content. Engineering Geology, 69, pp. 109-125.
Wang, F; Shibata, H. (2007). Influence of sol permeability on rainfall-induced flowslides in laboratory flume tests. Can. Geotech. Journal, 44, pp. 1128-1136.

Wieczorek, G.F.; Guzzetti, F. (2000). A review of rainfall thresholds for triggering landslides, Mediterranean Storms, Proceeding of the EGS Plinius Conference, Maratea, Italia.

\section{PARA CITAR ESTE ARTÍCULO TO REFERENCE THIS ARTICLE / PARA CITAR ESTE ARTIGO /}

Aristizábal, E.; Vélez, J.I.; Martínez, H. (2016). Influencia de la lluvia antecedente y la conductividad hidráulica en la ocurrencia de deslizamientos detonados por lluvias utilizando el modelo SHIA_LANDSLIDE. Revista EIA, 13(26), juliodiciembre, pp. 31-46. [Online]. Disponible en: https://doi. org/10.24050/reia.v13i26.863 